\title{
ESTABLISHING CORRELATIONS AND SCALE-UP FACTOR FOR ESTIMATING THE PETROLEUM BIODEGRADATION RATE IN SOIL
}

\author{
Alamgir A. Khan ${ }^{1}$; Richard G. Zytner ${ }^{2 *}$ and Zeny Feng ${ }^{3}$ \\ ${ }^{1}$ Post Doctorate Research Fellow, School of Engineering, \\ ${ }^{2 *}$ Professor, School of Engineering, \\ ${ }^{3}$ Assistant Professor, Department of Mathematics and Statistics, \\ University of Guelph, Guelph, ON N1G 2W1
}

*Corresponding author: Tel: +1-519-824-4120 Ext. 53859; Email: "rzytner@uoguelph.ca”

\section{Published Bioremediation Journal (2015) 19:32-46}

\begin{abstract}
The proper design of a bioremediation strategy for petroleum-contaminated sites requires a reasonable estimate of the biodegradation rate constant, which is not easy due to spatial heterogeneity. Accordingly, predictive models were developed by completing a bioventing study at the meso-scale. Reactors holding $4 \mathrm{~kg}$ of disturbed soil were tested using five different types of soils. Using statistical analysis, a two stage process was observed, with a fast rate of hydrocarbon degradation in the first $8 \mathrm{~d}$ and a slower rate in the remaining $22 \mathrm{~d}$. Review of the correlations showed that the initial population of petroleum degrading bacteria and increasing silt content had a positive effect on the degradation. A negative impact on the degradation rate was seen by increasing the fraction of organic matter and clay content. Comparison of previously completed micro-scale and meso-scale degradation rates gave a scale-up factor (SF) of $1.8 \pm 0.5$. Soils with an increased sand fraction had slightly higher SF values, whereas soils high in organic matter content had lower SF values. The measured SF values and developed correlations will help practitioners with site closure decisions, and indicate the need for additional SF work to allow better transfer of meso-scale data to the field.
\end{abstract}


Key words: TPH; hydrocarbon; soil remediation; bioventing; two stage degradation; petroleum contamination; correlation; regression models. 


\subsection{Introduction}

Soil contaminated with petroleum hydrocarbons is a global issue because of the potential human health risks from the carcinogenicity of some hydrocarbon compounds. Therefore, contaminated soil requires cleanup. An emerging remediation option is bioventing. Bioventing is an in-situ, cost effective technology (Khan et al., 2004), where indigenous microorganisms are stimulated by providing favorable conditions for aerobic biodegradation of contaminants (Leeson and Hinchee, 1996). This technology has been evaluated in the laboratory at the micro-scale (Moller et al., 1996; Zytner et al., 2001; Eyvazi and Zytner, 2009), and at medium or larger scale (Chang et al., 2011; Khan and Zytner, 2013) for the treatment of petroleum contaminated soils.

Besides the significant effect of soil physical-chemical properties on the biodegradation rate, limited work at the micro-scale (e.g., $200 \mathrm{~g}$ ) and meso-scale (e.g., $4 \mathrm{~kg}$ ) has been undertaken to relate the soil properties with the degradation rate constant. Moreover, understanding the scale dependent phenomena are the major challenges faced by the use of bioventing for successful implementation of laboratory data at the field scenario. Accordingly, the main objective of this study was to develop a regression model by correlating the degradation rate coefficients with the soil and biomass parameters from meso-scale bioventing experiments for different soils. These correlations will help estimate the degradation rate constants for various site conditions. Comparison of these rates to those previously obtained at the micro-scale (Eyvazi and Zytner, 2009), would permit a determination of the scale-up factor. Having the correlations and scale-up factor gives consultants useful tools to improve the assessment of site closure times.

\subsection{Overview}


The entry of petroleum hydrocarbons into soil is typically related to industrial spills, leaking underground storage tanks and leaking piping systems within the vadose zone (Lee et al., 2006; CCME, 2008; Eyvazi and Zytner, 2009). Once the petroleum hydrocarbons are in the soil, there is the concern about its migration into the groundwater. Bioventing is an in-situ treatment technology for gasoline contaminated soils, where bio-stimulation of native petroleum degrading bacteria make it a viable, cost-effective and less destructive technology for the aliphatic and aromatic hydrocarbons present (Khan et al., 2004; McCarthy et al., 2004; Lee et al., 2006; Chang et al., 2011).

Despite the very encouraging results of bioremediation at the micro-scale, there is no single degradation rate that represents a variety of site settings and types of soils ( Davis et al., 2003; Aichberger et al., 2005; Chang et al., 2011). In order to assist practitioners in making judgments, Brook et al. (2001) developed a correlation that related the degradation rate with nitrate and ammonia concentrations, while Zytner et al. (2001) related the degradation rate constant $(k)$ to $\mathrm{O}_{2}$ and temperature. Moreover, Eyvazi and Zytner (2009) further improved the correlation by including water contents and biomass into the model. Similarly, Jiang et al. (2011) conducted a field study and tried to evaluate the correlation of degradation rate coefficient with different petroleum compounds available in the soil.

Most of the literature studies have been carried out at the micro-scale, while limited literature exists on correlations at the larger scale. Not having these larger scale correlations makes it difficult to predict bioremediation behaviour, affecting planning decisions and the successful implementation of bioventing technology.

A recent study at the meso-scale showed that hydrocarbon degradation was a two stage process with a faster degradation rate in the initial eight days followed by a slower degradation rate for the remaining days of the $30 \mathrm{~d}$ treatments (Khan and Zytner, 2013). Degradation results measured at the meso-scale were compared with the rates determined through micro-scale correlation presented by Eyvazi and Zytner (2009). The comparison gave scale-up factors varying from 1.9 to 2.7 for the three types of soil considered. These differences in results between the meso and micro-scales show the importance of scale dependent factors when transferring laboratory 
results to the field. Accordingly, Khan and Zytner (2013) suggested that the Eyvazi and Zytner (2009) correlation be revised by incorporating the scale-up affects to better estimate the $k$ values for the range of soils studied. Accordingly, this paper studied two additional soils to provide data for a larger range of soil properties, which allowed for a thorough statistical analysis to be undertaken.

\subsection{Materials and Methodology}

Four types of soils having varied characteristics were collected from different research stations of the University of Guelph, ON. One soil was collected from the Elora Research station situated $21 \mathrm{~km}$ north-west of Guelph, with the second being from the Delhi Research station which is $120 \mathrm{~km}$ south of Guelph. Two other types of soils were collected from two different fields at the Ridge Town Research complex. Ridge Town is located around 200 km south-west of Guelph. A fifth type of soil was prepared by mixing an equal mass of Elora and Delhi soils.

All soils were air dried and then sifted by passing through a $2 \mathrm{~mm}$ sieve. Elora, Delhi and Mixed soils were similar to the soils used by Eyvazi and Zytner (2009). However, the two Ridge Town (RT) soils had slightly dissimilar properties mainly due to different texture. Soil classification and characterization was done by the Soil Laboratory at the University of Guelph Laboratory Services Division. The physical and chemical properties of the five soils are summarized in Table 1.

Medium scale reactors, named meso-scale reactors (MSR) were used in the bioventing experiments to better mimic the bioventing process taking place in the field (Khan and Zytner, 2013). Figure 1 gives a sketch showing principal parts of the meso-scale reactor.

A total of 18 experiments were conducted, with 6 replicates each for Delhi and Elora soils. Three replicates were done with Mixed soil, two with RT-1 and one confirmation experiment using RT-2 soil. Every single trial involved four reactors for measuring the decline of gasoline concentration in soil. For the all experiments excluding RT-2, two extra reactors were added for biotic and abiotic control.

As the soil was stored dry in bags, preliminary testing showed that the microorganisms were in the inactive state. Reactivation of the biomass was enhanced by adding to the soil nutrients and 
water several days before the start of each experiment. Ammonium Chloride $\left(\mathrm{NH}_{4} \mathrm{Cl}\right)$ was used as the source of nitrogen with synthetic gasoline as the carbon source to achieve a C:N ratio of 10:1 (Eyvazi and Zytner, 2009), which was shown to be sufficient. The desired soil water contents, 50 to $70 \%$ of soils field capacity were maintained in the soil during the treatment period. A Hydrosense reflecto meter (Campbell Scientific ${ }^{\circledR}$ ) was used for frequent monitoring of the soil water content.

Synthetic gasoline at a concentration of $4000 \mathrm{mg} / \mathrm{kg}$ was added to the soil in a closable container and then mixed manually for about fifteen to twenty minutes. The composition of the synthetic gasoline by mass was 3.2 \% Naphthalene, $11.9 \%$ 1,3,5-Trimethylbenzene, $23.9 \%$ mXylene, 36 \% Toluene and 24.9 \% Isooctane (Shewfelt et al., 2005; Eyvazi and Zytner, 2009; Khan and Zytner, 2013).

The key parameters monitored during the treatments were concentration of total petroleum hydrocarbon (TPH) in the soil, air flow rates, volatilization loss of gasoline and concentration of oxygen in the off gases. Soil $\mathrm{pH}$, soil water content, room temperature and population of petroleum utilizing bacteria were also monitored (Khan and Zytner, 2013).

The air flow rate of $0.42 \mathrm{ml} / \mathrm{min}$ was achieved by drawing air through industrial hygiene pumps (Gilian, model: LFS-113DC). Air flow meters (Gilmont Micro Flow Meter, Model: GF-9760) were used to calibrate the pumps. Figure 2 shows the experimental system in the fume-hood, along with associated equipment such as: oxygen meter, reflectometer, suction pumps, air flow meter, and MSRs.

The amount of hydrocarbon volatilization was determined by carbon sorbent tubes (Supelco $\mathrm{ORBO}^{\circledR} 32$ Small) which were installed in the suction line. Likewise, the oxygen level in the off gases was monitored by using flow-through head (Model AO-002) of the Apogee Oxygen Meter (Model: MO-200 Series) (Khan and Zytner, 2013).

Biotic and abiotic control reactors were set up in sequence with the experimental reactors. It was expected that a variation in the population of petroleum degrading bacteria in soil would directly relate with the gasoline contamination and its breakdown. Biotic control was run in order to see the difference in microbial population where all conditions were the same except for the use of clean soil. 
Similarly, a small initial population of petroleum degrading bacteria in contaminated soil was likely to degrade gasoline at a very low rate (Eyvazi and Zytner, 2009). In view of that, an abiotic control was run parallel to the experimental reactors to test the decline of gasoline concentration. In the abiotic control, the initial population of bacteria was reduced where soil was spiked with the same concentration of hydrocarbon. The biotic control was the same as the experimental reactors except the addition of gasoline. However, the abiotic control was prepared by sterilizing soil through a dry heat method (Wolf and Skipper, 1994; Alef and Nanniperi, 1995; Trevors, 1996; Khan and Zytner, 2013).

The population of petroleum degrading bacteria (PDB) and total heterotrophic bacteria (THB) were evaluated using the spread plating technique previously reported in Shewfelt et al., (2005) and Eyvazi and Zytner (2009). In this technique, Bushnell-Hass (BH) culture medium was prepared and placed in Petri plates along with gasoline at a dose of $4 \mathrm{~g} / \mathrm{L}$ as the carbon source. To test the soil, 5 g soil was collected with sterile tools and placed in $45 \mathrm{~mL}$ of sterile sodium pyrophosphate solution and shaken for about $1 \mathrm{~h}$ at $200 \mathrm{rpm}$ to separate the attached microorganisms from the soil. The suspension was then serially diluted. From each dilution, $0.1 \mathrm{~mL}$ of suspension was spread on the $\mathrm{BH}$ medium. The colonies were counted after 7 and 14 days of incubation at $25 \circ \mathrm{C} \pm 1 \circ \mathrm{C}$.

Methylene chloride was used as the solvent to extract gasoline from both soil and sorbent tube samples. A gas chromatograph (GC) equipped with a DB5 capillary column from J \& W Scientific (dia. $0.32 \mathrm{~mm}$, length $30 \mathrm{~m}$ and film thickness of $1 \mu \mathrm{m}$ ) and a flame ionization detector (Hewlett-Packard 5890 Series II) was used to analyze total petroleum hydrocarbons extracted from soil and sorbent tube samples. The system was equipped with an auto sampler, Hewlett Packard (HP 7673). The oven and detector temperature was set at $300^{\circ} \mathrm{C}$, with an oven ramp program that had an initial temperature of $40^{\circ} \mathrm{C}$ for $2 \mathrm{~min}$. followed by a $5^{\circ} \mathrm{C} / \mathrm{min}$ ramp rate to a temperature of $100^{\circ} \mathrm{C}$. The temperature then increased up to $250^{\circ} \mathrm{C}$ at $10^{\circ} \mathrm{C} / \mathrm{min}$ rate and was held at this temperature for $3 \mathrm{~min}$. The total run time was $32 \mathrm{~min}$. 


\subsection{Statistical Methods}

The collected data was analyzed using graphical and statistical methods. Computer software such as: SYSTAT (Eyvazi and Zytner, 2009; SYSTAT, 1992), R (R, 2013) and Microsoft Office Excel (MSE) (MSE, 2007) were used in the data analysis. $R$ is free software that is very popular software for statistical analysis. Statistical analysis was started by using MSE in which the Pearson correlation coefficient test was conducted. After reviewing the Pearson coefficients to identify which predictive variables should be included in the model, the two way stepwise regression analysis was initiated based on the procedure first proposed by Efroymson (1960).

The selected predictive variables were used in constructing draft correlations. The draft correlations were then checked using a sensitivity test. A manual sensitivity test was conducted by changing the value of the explanatory variables while keeping other variables constant and observing the effect on the dependent parameter (degradation rate constant).

Comparative evaluation of the correlation developed by Eyvazi and Zytner (2009) at the micro-scale with the developed Stage-2 correlation assisted with determining scale-up factors. Equation 1 displays the Eyvazi and Zytner (2009) micro-scale correlation:

$$
\begin{aligned}
\ln k_{E} & =2.803 \times \mathrm{PDB}+0.21 \times \text { Sand }+4.886 \times \mathrm{OM}-0.094 \times \text { Clay }-0.004 \times(\mathrm{SW} \times \mathrm{Sand}) \\
& -0.021 \times(\text { Sand } \times \mathrm{PDB})-0.632 \times(\mathrm{OM} \times \mathrm{PDB})+0.004 \times(\mathrm{SW} \times \mathrm{Clay})-23.5
\end{aligned}
$$

Where:

$$
\begin{aligned}
& K_{E}= \text { first order degradation rate, } \mathrm{d}^{-1} \\
& \text { PDB = } \text { initial population of petroleum degrader microorganisms in soil, log } \\
& \text { colony forming units (CFU/g of soil) } \\
& \text { Sand = sand content, } \% \text { Clay = clay content, } \% \\
& \text { SW = soil water content, } \% \\
& \text { OM = organic matter content, } \%
\end{aligned}
$$


Values of soil and biomass variables were plugged into Equation 1 to assess the micro-scale degradation rate constant, referred to as Eyvazi's $k\left(k_{\mathrm{E}}\right)$. Similarly, the newly developed correlation for second stage degradation was used to estimate $k_{s t g 2-P}$, where, $k_{s t g 2-P}$ is the predicted value of degradation rate constant for Stage-2. The ratio of $k_{S t g 2-P}$ and $k_{E}\left(\right.$ i.e., $\left.k_{S t g 2-p} / k_{E}\right)$ gives the estimated value of scale-up factor (SF) for each type of soil.

\subsection{Results and Discussion}

Previous studies suggested that the data should be broken into two groups or analyzed with a more complex model (Shewfelt et al., 2005; Zytner et al., 2006; Maletić et al., 2009; Khan and Zytner, 2013). Hence, the data recorded through experiments was initially analysed to verify the two stage degradation trend and then improved with two additional soils to increase the application range of the correlations and provided additional points for a thorough statistical analysis. This is followed by the analysis and development of stage-wise correlation models, and then the scale-up factor.

\subsection{Two Stage Degradation}

The observed soil hydrocarbon data was analyzed using semi- $\log _{\mathrm{e}}$, where the degradation rate of hydrocarbon in soil was determined by plotting $\log _{\mathrm{e}}$ of the gasoline concentration versus time. The resulting slope, or coefficient of time in the exponent was taken as the degradation rate constant $(k)$.

Previous analysis done by Khan and Zytner (2013) utilizing Delhi, Elora and Mixed soils with similar experimental settings, found that the biodegradation of hydrocarbon in the meso-scale was a two stage process with a faster rate during the first 8 to 11 days. Khan and Zytner (2013) introduced d8 to $\mathrm{d} 11$ as the transition period during which the degradation transferred from Stage-1 to Stage- 2 .

In order to determine the appropriate transition day from Stage-1 to Stage-2, the inflection points of all the degradation curves for each experiment were analyzed. The analysis gave an average value of the transition day as $8.4 \mathrm{~d}$ for Delhi soil, $8.0 \mathrm{~d}$ for Mixed soil and $8.5 \mathrm{~d}$ for Elora soil. 
To confirm this two stage trend, two additional experiments were completed using RT-1 soil and one additional treatment with RT-2 soil. Figure 3 displays the pooled results for the two stage degradation trend for RT-1 soil. Similarly, Figure 4 displays the two stage degradation trend for RT-2. The average inflection point from Stage- 1 to Stage-2 for both the RT soils was $8.3 \mathrm{~d}$.

In order to find statistical support for the selected $8 \mathrm{~d}$ transition point, analysis of all 18 inflection points from all the experiments completed with the 5 soils was done. The result was an average transition time of $8.4 \pm 0.6 \mathrm{~d}$, which confirms that $\mathrm{d} 8$ was a reasonable transition point from Stage-1 degradation to Stage-2 degradation. Moving forward, all data sets were split into two groups for analysis, with Stage-1 referring to d0-d8 and Stage-2 referring to d8-d30.

In addition to the degradation trends observed in Figures 3 and 4, there was also a fast increase in the microbial population in the first eight days, followed by little change in PDB population. Khan and Zytner (2013) reported that the highest population of petroleum degrading bacteria was between $\mathrm{d} 7$-d8, followed by minimal variation in PDB for the remaining period of treatment. As such, Stage-1 can be considered the time where the ideal conditions allow the dormant microbes to become highly active due to plenty of food and nutrients present in the soil. Stage- 2 is then the time where the microbes attain a normal population, providing a rate of hydrocarbon degradation that approaches a realistic and balanced state. The balanced state is defined as the second stage of degradation in which microbial function was continuous and stable for the remaining 22 days.

\subsubsection{Degradation Rate}

Table 2 summarizes the experimental values of the first order degradation rate constants for both stages for the five different tested soils. The observed value of the degradation rate constant at

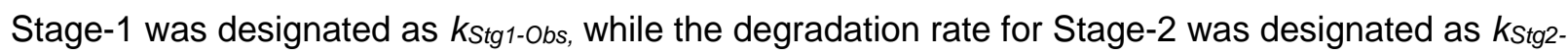

obs. Also given in Table 2 is the overall r-squared values for each determined degradation rate, 
along with how many data points were used. The various values show that the quality of data is good and repeatable.

The degradation rate constant for each soil was measured by regressing the combined data of the various treatments completed for that soil. The exponential trend lines fitting the data for d0-d8 and $d 8-d 30$ represented the trend of pooled data for each distinctive soil. The previously reported first order degradation rate constants for Stage- 1 were $0.60 \mathrm{~d}^{-1}$ for Delhi, $0.48 \mathrm{~d}^{-1}$ for Mixed soil and $0.46 \mathrm{~d}^{-1}$ for Elora (Khan and Zytner, 2013), while for the current study, values of $0.41 \mathrm{~d}^{-1}$ for RT-1 and $0.44 \mathrm{~d}^{-1}$ for RT-2 were also determined. Likewise, the regressed degradation rate constant reported for Stage-2 were $0.12 d^{-1}$ for Delhi, $0.09 d^{-1}$ for Mixed soil, $0.08 d^{-1}$ for Elora, $0.04 d^{-1}$ for RT-1 and $0.04 \mathrm{~d}^{-1}$ for RT-2 soil.

The highest rate of degradation measured was for Delhi soil, while the lowest rate of degradation was obtained for RT-1. Composition of the soil suggests that parameters such as cation exchange capacity (CEC), OM, PDB and soil texture are important factors in controlling the degradation rates. Delhi soil had the highest sand fraction at $85 \%$, while sand content in RT-1 was only $56 \%$. Organic matter was $1.1 \%$ in Delhi and $3.5 \%$ in RT-1. The initial population of petroleum degrading bacteria was 6.1 CFU/g in Delhi soil and 6.6 CFU/g in RT-1 soil. Similarly, the CEC in Delhi soil was $4.9 \mathrm{cmol} / \mathrm{kg}$ of soil, while CEC in RT-1 was $15.2 \mathrm{cmol} / \mathrm{kg}$.

The larger fraction of sand in the Delhi soil along with the larger pore size and sufficient water can facilitate the mobility of microbes. Conversely, higher clay contents, $17.1 \%$ in RT-1 as compared to $5.3 \%$ clay in Delhi soil might have reduced the pore size, affecting the mobility of microbes and their access to food in RT-1. This also shows that a larger ratio of volume of soil/surface area provided a better environment for microbial activity in soil. As such, the microorganisms obtained better access to both carbon and nitrogen sources of food in Delhi soil, resulting in a higher degradation rate. Results also revealed that a larger fraction of OM caused a major hindrance to the rate of biodegradation and this parameter will be discussed further later on.

Overall, higher rates of degradation were determined on the larger scales when compared with micro-scale results. The negligible volatilization of gasoline reported by Khan and Zytner (2013) 
as $0.03 \%$ of the initial gasoline concentration through two a stage carbon sorption system and the rapid increase of microbial population demonstrated better aerobic degradation conditions at the meso-scale. This was possible by the good aerobic setting where the oxygen content in the soil remained at $18-20 \%$ and represents real site conditions. The current findings strengthen the importance of using two stage correlation models when evaluating the degradation of gasoline at field scale.

\subsection{Correlations Analysis}

The correlation analysis was carried out by using the results from all the experiments. The observed data was broken into Stage-1 and Stage-2 groups as discussed in Section 4.1 and regressed accordingly.

\subsubsection{Stage-1 Correlation}

The values of the degradation rates for Stage-1 (kstg1-obs), were plotted against the corresponding fractions of soil texture, organic matter, soil $\mathrm{pH}$, and initial population of petroleum degrading bacteria (PDB). Figure 5 shows a gradual increase in the degradation rate with an increase in soil sand content, while the value of $k_{\text {stg1-obs }}$ decreased with an increase in values for the remaining five variables. Moreover, the high values of coefficients of determination for clay $\left(R^{2}=0.74\right)$, organic matter $\left(R^{2}=0.81\right)$ and $p H\left(R^{2}=0.81\right)$ suggested a considerable relationship of these three variables with kstg1-obs. The individual relationships of the remaining parameters appeared to be less significant.

The increasing trend of hydrocarbon degradation with the increment of sandy fraction may be due to larger pore size in the soil column and better availability of oxygen for aerobic microbial activity. However, the decline of the degradation rate constant with the increase in initial population of petroleum degradation bacteria and very low $R^{2}$ value of 0.45 was expected as discussed earlier in Section 4.1 for the comparison of Delhi and RT-1 soils. The decline of $k_{\text {stg1-Obs }}$ with increasing PDB could be linked with the higher initial population of PDB in Elora and Ridge Town soils. 
Figure 5 shows the good relationships of the individual variables with the degradation rate constant ( $k_{\text {stg1-obs }}$. However, these results cannot be generalized without evaluating the combined impact of the input parameters on $k_{\text {stg1-obs }}$ values. Therefore, further data analysis was carried out by using a Pearson correlation test (MSE, 2007) to investigate the correlation between $k_{\text {stg1-obs }}$ and input parameters. The square matrix given in Table 3 displays the relationship between the degradation rate constant $k_{\text {stg1-obs }}$ and other covariate factors. The results show a positive correlation between the degradation rate and sand content while all other factors demonstrated a negative relation with $k$ stg1-obs.

Further review of the Pearson coefficients in Table 3 show that the model expressed a multicollinearity problem, where a strong correlation exists between sand, $\mathrm{pH}, \mathrm{CEC}$ and $\mathrm{SW}$. Three fractions of soil (sand, silt and clay) are naturally interconnected as their sum always makes $100 \%$. This kind of multicollinearity can lead to imprecise estimates of coefficients. As such, the removal of strong inter-correlated variables can reduce multicollinearity significantly. Sand, silt, clay and CEC all displayed strong correlation with the majority of the covariates as compared to their relationship with the response variable (kstg1-obs). For example, the Pearson coefficient for the relation of sand with kstg1-Obs was 0.72 . However, sand displayed a strong correlation with most other covariates, as all the Pearson coefficients for all covariates exceeded 0.72 except for PDB which was 0.44. A similar problem was observed for silt, clay and CEC. The Pearson coefficients for the relation of CEC with kstg1-obs was 0.78 , while CEC was strongly related with most other covariates as the Pearson coefficient was higher than 0.78, except for PDB which was 0.52 .

Soil fraction and CEC cannot be controlled during the bioventing operation. However, the removal of CEC and one of the soil fractions from the predicting variables, for example sand, can lessen the influence of multicollinearity. Both $\mathrm{SW}$ and $\mathrm{pH}$ faced a similar issue. However, these parameters can be controlled during experiments; so, SW and $\mathrm{pH}$ were retained for further stepwise testing for their significance to the model. The variables such as PDB and OM were not omitted from the regression analysis since the intensity of their multicollinearity was less than the removed variables. For example, the Pearson coefficient for the correlation of OM with kstg1-obs was 0.9, which 
was higher than most variables except three, clay, CEC and SW. Since it was already decided to omit CEC, only two covariates were problematic when comparing all six with sand and CEC, suggesting the removal of SW. Thus, silt, clay, OM and PDB were kept as input parameters for further analysis. The parameter $\mathrm{pH}$ was also removed as it had a minimal impact on the degradation rate (small Pearson coefficient in Table 3), plus only a small range of pH was studied, 6 to 8.

After failing with the linear stepwise regression technique due to negative $k$ values, and a power analyses, stepwise regression model using natural $\log _{\mathrm{e}}(\mathrm{In})$ to transform $k$ stg1-obs was considered, which ensured a degradation rate $k$ above zero. The form of the Ink model is given in Equation 2:

$\ln k=X \beta+\varepsilon$

where:

$k \quad=$ degradation rate constant $\left(\mathrm{d}^{-1}\right)$

$\mathrm{X} \quad=$ any input variable

$\beta=$ coefficient of variables

$\varepsilon \quad=$ intercept of the slope for linear equation

The regression analysis showed that silt, clay, OM and PDB values all correlated with Ink at least at a significant level of $5 \%$. Table 4 gives the coefficients for the significant factors and the associated standard errors, and the p-values of these estimates. The coefficients of silt and OM both had three stars under significant codes, which indicates these parameters are highly significant at less than 0.001 , meaning that the chance of variation in the estimate of $\ln k$ is $0.1 \%$. This is seen by the $\mathrm{p}$-value of 0.0004 for silt and 0.0001 for OM. Likewise, the $\mathrm{p}$-value for PDB is 0.0034 , and has been given two stars as the significant code, which suggest a $1 \%$ level of significance. The p-value for clay is 0.0419 , given a single star, which shows that the level of significance is $5 \%$. In short, the correlation of all the selected parameters with Ink possess $95 \%$ confidence level or better for 
accuracy and that silt, clay, OM and PDB are important factors. Equation 3 describes the final fitted model for Stage-1:

Inkstg1-P $=0.0116 \times$ Silt $-0.0191 \times$ Clay $-0.3168 \times$ OM $+0.6618 \times$ PDB -4.166

Figure 6 shows the plot of the fitted Inkstg1-P (solid line) given by Equation 3 against the observed Inkstg1-obs data. Both sets of data, predicted (Inkstg1-P) and observed (Inkstg1-obs) compare well, with a high value of $\mathrm{R}^{2}$ at 0.93 .

Sensitivity of the explanatory variables was manually evaluated by increasing the value of one of the explanatory variables and holding the others constant. For example, while holding clay, OM and PDB as constants, and increasing the silt fraction by one unit, Inkstg1-p increased by 0.012 units. With $\log _{\mathrm{e}}$ being used, the degradation rate $k_{\text {stg1-P }}$ will increase by exp 1.012 fold. The result indicates that the newly developed correlation for Stage-1 is sensitive to minor variation in the explanatory variables. The most sensitive variable was PDB, because it has the largest value of the coefficient in the Equation 3. Taking Delhi soil as an example and increasing initial population of PDB by 0.1 unit (6.1 CFU/g to 6.2 CFU /g of soil) and holding clay, OM and silt as constants, Inkstg1-P increased by 0.066 units. The degradation rate $k_{s t g 1-P}$ will increase by 1.066 fold. A sensitive response of the initial population of PDB with a favorable environment makes sense, because a higher initial population of microorganisms needs more food resulting in higher rates of hydrocarbon consumption.

In the light of the above facts and analysis, Equation 3 is recommended as the optimum correlation for the reliable prediction of degradation rate constant $(K \operatorname{stg} 1-P)$ for the first eight days of hydrocarbon degradation in the soil. Summarizing the findings reveals that increasing Silt count and PDB have a positive impact on $k_{S t g 1-P}$, while clay and organic matter exhibited a negative impact. The positive impact of silt can be related to a reduction in surface area of soil particles and increase of pore size, increasing conductivity of oxygen in the soil. 


\subsubsection{Stage-2 Correlation}

All the steps followed to determine the Stage-1 model were used to develop the Stage-2 model. Plots of the individual variables against $k_{\text {stg2-obs }}$ values were very similar to the plots shown in Figure 5. Table 5 gives the corresponding Pearson correlation coefficient test values and shows that the degradation constant has a positive relationship with sand content, while a negative impact exists for all the remaining parameters when analyzed with respect to $k_{\text {stg2-obs. }}$ It should also be noted that initial PDB considered for Stage-2 was the population of petroleum degrading bacteria measured on d8 of the treatment period.

Table 5 again shows the multicollinearity problem that was seen in the Stage- 1 analysis, where a strong correlation exists between sand, silt, $\mathrm{PDB}, \mathrm{pH}, \mathrm{CEC}$ and $\mathrm{SW}$. The soil factions have a strong natural correlation and were considered responsible for the overall collinearity issue. As such, sand and CEC were removed from further regression attempts as they were the most problematic for multicollinearity, where the Pearson coefficients were -0.723 for CEC and -0.653 for sand.

The core variable, PDB also exhibited a collinearity problem but it was kept in the input variable for further testing due to its significance as predicting variable. SW was retained because it can be controlled during treatments, while $\mathrm{pH}$ was again removed due to the narrow range in which it exists. The parameter OM was not omitted from the regression analysis since the intensity of its multicollinearity was less than the removed variables. For example, Pearson coefficient for the correlation of OM with $k$ stg2-obs was 0.891 which was higher than the most variables with the exception of three covariates, clay, CEC and SW. Thus, the variables retained for further regression analysis were silt, clay, OM, PDB8 and SW.

As in the Stage-1 analysis, stepwise regression using Equation 2 was applied. The stepwise regression using SYSTAT and R software gave a model at a significant level of $5 \%$ or better for the variables Silt, Clay, OM, and PDB. Table 6 summarizes the estimated model coefficients and the corresponding levels of significance (significant codes). Even though SW was seen as non- 
significant, it was still retained in the correlation as removal made the model unstable. The resulting Stage-2 model is given by Equation 4:

Inkstg2-P $=0.0231 \times$ Silt $-0.0355 \times$ Clay $-0.383 \times$ OM $+0.825 \times \mathrm{PDB}_{8}-0.048 \times \mathrm{SW}-6.774$

Figure 7 displays the fitted Inkstg2-p as estimated through Equation 4 against the observed Inkstg2-obs values. It is seen that Inkstg2-obs and the predicted Inkstg2-P values are close to the straight line with a high value of $R^{2}=0.94$, supporting the recommendation that Equation 4 is an optimum correlation to estimate the degradation rate constants for Stage-2.

Review of Equation 4 indicates that there are negative effects of increasing organic matter upon $k_{S t g 2-p}$ and a strong positive impact on the initial population of petroleum degrading bacteria. Similarly, an increase in the soil water content displayed a negative relation with degradation rate constant. For the experiments completed, SW was kept in the range of 50-70\% of soil water holding capacity. This finding applies to both the Elora and RT soils, where soil having high water holding capacity showed lower rates of degradation as compared to the Delhi soil, which has a lower SW capacity. This wide range of SW capacity for the soils tested, probably contributes as to reason why this parameter was rated as insignificant during the stepwise regression process.

Sensitivity testing showed that Equation 4 is also sensitive to minor changes in the explanatory variables. For example, a 1 unit increase in silt value increased the $\ln k_{\text {stg2-p }}$ by 0.023 . With $\log _{e}$ being used, the degradation rate kstg2-P will increase by exp (0.023), or 1.023 fold. Analogous to Equation 3, the outcome of Equation 4 also indicates that the newly developed correlation for Stage- 2 is highly sensitive to a minor variation in the explanatory variables. The most sensitive variable was $\mathrm{PDB}_{8}$ because in Equation 4, $\mathrm{PDB}_{8}$ has the largest value of the coefficient (0.825). Using Mixed soil as an example; while holding silt, clay and OM as constants, and increasing the $\mathrm{PDB}_{8}$ by only 0.1 units (i.e., 6.8 to $6.9 \mathrm{CFU} / \mathrm{g}$ of soil), Inkstg2-P increased by 0.083 units. With $\log _{\mathrm{e}}$ being used, the degradation rate $k_{\text {stg2-P }}$ will increase by 1.086 fold. A sensitive 
response of the $\mathrm{PDB} 8$ with a favorable environment makes sense, because even a slight increase in $\mathrm{PDB}_{8}$ means more potential to reach and consume hydrocarbon as food.

The coefficient value for $\mathrm{PDB}_{8}$ (0.825) for Stage-2 in Equation 4 was larger than the coefficient of PDB (0.662) for Stage-1 given in Equation 3. This was related with the stability of microbial population on d8 and onward. In general, during meso-scale treatments, the population of petroleum degrading bacteria increased from $\mathrm{d} 0$ to $\mathrm{d} 8$, while the population remained almost stable from d8 to d30. For that reason, a minor increase in PDB8 can increase the degradability of the system.

Further review of Equation 3 and 4 shows similarities in the variables, which necessitates further discussion. The positive effect of increasing silt content and the negative relation of increasing clay content, suggest an influence of soil aggregates on the rate of biodegradation. This also shows that biodegradation in soil is not only dependent on the activity of microbial biomass, but also on microbial mobilization within the soil aggregates. Soils with a higher clay fraction have larger surface area and smaller pores which might trap the microbes, giving them less access to the contamination. On the other hand, an increase in silt content can be easier on air flow, which provides a better environment for the biomass. Amellal et al. (2001) tested the relationship of soil fraction with microbial population for the degradation of petroleum hydrocarbons. Intermediate aggregates of soil contained the highest population of PDB when Amellal et al. compared PDB within the finest and the coarsest aggregates of soil. Accordingly, the results of Amellal et al. (2001) support the findings of the current study, which suggests a faster break down of hydrocarbon in soils with increasing silt fraction as compared to the soils with increasing clay fraction.

The negative impact that organic matter had on the degradation rate was seen in all soils studied. In general, for the soils studied, higher organic matter contents were associated with increasing clay fractions. The combination of tiny pores associated with a higher clay fraction and an increased amount of organic matter content (OM) might have reduced the air movement in soil. Less air movement can cause a shortage of oxygen in particular spots of the soil, affecting aerobic activity. Yang et al. (2011) noticed lower microbial activity in soils with both the lowest and the 
highest level of organic matter due to nutrition limitation and petroleum sequestration. The current study did not directly support this finding, which is related to sufficient nutrients being added to the soil in this study.

Moreover, organic matter might have served as an alternate food source for the microbes (Guo et al., 2012) resulting in a decrease in the degradation of hydrocarbon as compared to soils having lower organic matter contents. Broadly speaking, another possibility for negative connection of OM with $k$ can be sorption. Sorption of gasoline into organic matter and clay might have attributed to substrate limitation (Labud et al., 2007). Due to the transformation or mineralization of contaminants, enzymes of microbial cells need to get direct access to the compound (Bressler and Gray, 2003). Sorption of contaminant onto the soil constituents reduces the aqueous and gaseous phase concentrations (El-Tarabily, 2002; Harper et al., 2003) and might cause substrate limitation in soils which have higher organic matter fractions. Conversely, Del'Arco and de Franca (2001) discussed that sorption helped enhance the contact between the contaminant and the microorganism when the contaminant concentration is very low, eliminating the substrate limitation problem. However, the negative impact of organic matter on the degradation rate constant also agrees with the conclusions made by several other researchers (Eyvazi and Zytner, 2009; Harvey et al., 1986; Scully et al., 2003).

Review of the parameters in the regression models, showed that petroleum degraders are the core factor of biodegradation as the relationship was positive with degradation rate constant. This contradicts the findings of Figure 5, where an increase in PDB appeared to be negatively related with kstg2-obs. The microorganisms are extremely sensitive and their population is highly reliant on the environmental conditions. A minor variation in the ecological and environmental conditions can influence growth and activity, and affecting biodegradation rates (Eyvazi and Zytner 2009). Similar variation with minute differences in the environmental factors was seen in the PDB for different treatments and this was related to the sensitivity of microbial response.

Both correlations suggest that PDBs are a dominant factor. The initial population of petroleum degrading bacteria increased considerably during Stage-1, while small changes in the 
microbial number were noticed during the remaining days of the experiments. The average initial population of petroleum degrading bacteria was $6.1 \mathrm{cfu} / \mathrm{g}$ of soil for Delhi soil, 6.2 for Elora, $6.1 \mathrm{for}$ Mixed, 6.6 for RT 1 and $6.6 \mathrm{cfu} / \mathrm{g}$ of soil for RT-2. The major increase in the population of petroleum degraders was seen before transition in the degradation rate from Stage-1 to Stage-2. The asymmetric increase of PDB was seen in different soils from d0 to d8 such as: $13 \%$ increase in Delhi, $10 \%$ in Elora, $11 \%$ in Mixed, $4.5 \%$ in RT-1 and $6.7 \%$ in RT-2 soil. This asymmetrical increase in PDBs in dissimilar soils did not appear to decrease the dominancy of PDB in the second stage regression model.

Given the lower initial count and higher rate of increase in the population of PDB for Delhi soil when compared to the RT-1 soil, the microbial population for Delhi and RT-1 reached a comparable level on d8. In analogy to Stage-1, the degradation rate for Delhi soil was still higher than RT-1 even for Stage-2. At this point, the higher PDB for Delhi soil came up as stronger factor because the values for the remaining input parameters were similar for both the stages. In other words, the fitting parameters for the second stage were similar to the variables for the first stage except for varied values of PDB. Conditions and results for the remaining three soils, Elora, Mixed, and RT-2 were also similar. Moreover, a higher initial population of petroleum degraders in RT-1 on d0, with little increase in their population during the period of treatment also support smaller $k$ values for RT-1.

\subsection{Scale-up Factor (SF)}

Given the optimum experimental conditions which included successful stimulation of the microbial population to degrade the synthetic gasoline and minimal volatilization, the difference in degradation rates between the micro and meso scale is believed to be mainly ascribed to scale-up factors (Khan and Zytner, 2013). Scale-up factors include microorganism population, availability of nutrients and substrate, easy access to oxygen, adsorption/desorption, inter-phase transfer, soil type, soil heterogeneity, water contents and weathering of contaminant (Ko et al., 2007). The existence of scale dependent rates emphasizes the need for correctly adjusting respirometer data from degradation assessment studies before transferring the results to the field. The parameter for 
doing so is called the scale-up factor (SF). This study evaluated SF for micro to meso-scale, and further the work is required for meso-scale to field scale. The current work is the starting point.

The scale-up factor was calculated by dividing $k_{E}$ by $k_{S t g 2-P}$ as both represent the long term degradation that develops at a remediation site (Khan and Zytner, 2013). The specific degradation rates for $k_{E}$ were calculated using Equation 1, while $k_{S t g 2-P}$ was calculated using Equation 4 . For the soils tested, Delhi, Elora, Mixed, RT-1 and RT-2 the corresponding scale-up factors were 2.6, 1.9, $1.9,1.1$, and 1.7 respectively. Taking the average for all soils tested gives an SF of $1.8 \pm 0.5$ for experiments conducted with disturbed soil.

These scale-up factors showed that the increase in volume of soil in the micro-reactors increased the rate of hydrocarbon degradation. Ko et al. (2007) also found increased biodegradation with scale-up process from batch (small) through pilot (medium) to large or field-scale land farming. During their study, Ko et al. (2007) reported some inhibitory factors such as: lower concentration of oxygen, poor diffusion of chemicals, and smaller initial population of biomass. It was also noted that the biodegradation rate was a function of diffusion phenomena related with microbial activity. This is where volume of soil, scale of treatment, distance travelled by air or chemicals come into play. In other words, the diffusion of oxygen and chemicals can strongly be related with volume of soil and distance traveled by the flowing air from point of entry to the point of exit. This finding suggests, diffusion can be a major factor directly affecting the scale-up of degradation at soil remediation sites. For the current experimental setup with disturbed soil, diffusion was improved by the gradual radial flow air taking place as compared to the micro-scale set-up in Eyvazi and Zytner (2009).

Review of the data showed that the increase in organic matter and clay fraction showed a noticeable decrease in SF. At the micro-scale study level, due to size of the apparatus, the soil most likely did not have the same density as the field condition and could not exhibit the response/effect of the variables as prominently as the meso-scale. Similarly, an increase in PDB and silt fraction also showed a decrease in SF but this decrease is not as sharp, indicating that the microbial mass and silt were equally influential in both scales. Accordingly, the largest value of SF (2.6) for Delhi soil among all soils can be related to the highest fraction of sand. Likewise the smallest value of SF for 
RT-1 might have appeared due to the largest contents of organic matter in RT-1. The moderate combination of parameters in remaining three soils exhibit SF close to 2.

\subsection{Application of Correlations}

Consultants or site managers for remediation projects are always interested in the careful investigation of technologies prior to the selection of the best option to remediate the site. The estimation of remediation cost and time for site closure are the major challenges, where reliable assessment of degradation rates can help determine the time of site remediation and then subsequently the cost. Normally, the determination of degradation rates need time-consuming field or laboratory testing. However, the newly developed two stage correlations provide an improved means of estimating the degradation rates for hydrocarbons in soil. The parameters in the correlations are simple and easily measured through straightforward laboratory analysis, which makes it a very economical option as compared to a full blown lab study. Special care must be undertaken for selection of the sampling point so that samples are representative of the whole contaminated site.

Once the requisite variables are available, the required degradation rates can be determined. These values can then be used to predict time for site closure. The rate coefficients can also be placed into more complex computer models, as they would be representative of the site, further improving predictions. Additional improvement of the model predictions would be made by incorporating SF. The current study shows the importance of SFs and confirms that more work is needed to better understand how meso-scale results can be transferred to the field scale results. The specific areas of research needed include working with undisturbed soil, to evaluate how disturbed soil influences soil heterogeneity, which ultimately impacts the scale-up factor. Then there are the challenging factors like uniform distribution of the nutrients and water, important elements for microbial activity. Studying these factors will provide for improved SF values and a better understanding on how best to transfer laboratory results to the field. 


\subsection{Conclusions}

The results from the current study showed that the biodegradation rate of hydrocarbons in soil can be reasonably estimated a two stage system using soil and biomass parameters. Correlations were determined by conducting experiments at the meso-scale with disturbed soil, which provided an improved representation of field conditions. The developed first stage correlation (Equation 3) for the starting eight days of degradation successfully predicted the degradation rate constants for the soils tested with $\mathrm{R}^{2}$ of 0.93 . Likewise, the second stage correlation with high value of $R^{2}$ of 0.94 also showed that the final model (Equation 4) also fitted the data. Both regression models displayed a strong positive relationship of silt and PDB with the degradation rate constant, while organic matter and clay showed negative relation with $k$. Among other variables, the initial population of petroleum degraders appeared as a major factor affecting the biodegradation rate of gasoline in soil.

Comparison of results from the micro-scale (Equation 1) and meso-scale (Equation 4) correlations suggested that the scale-up factor (SF) ranged from 1.1 to 2.6 depending upon the type of soil. The average SF value was $1.8 \pm 0.5$, showing a reasonable spread of all the soils tested. The smallest value of SF (1.1) was related to the negative impact of increasing OM as in RT-1. The largest SF (2.6) was for Delhi soil having high sandy fraction, which was reasonable. Further work is needed to evaluate how scale-up factors behave when transferring meso-scale results to the field.

The resulting empirical correlations provide an easy means of assessing degradation potential, minimizing the need for time-consuming laboratory studies. Appropriate estimation of degradation rates will allow consultants or site managers to carefully consider bioremediation technologies and select the best option for site remediation.

\section{Acknowledgments}

Research support was provided by a Canadian NSERC Discovery Grant.

\section{References}


Aichberger, H., Hasinger, M., Braun, R. and Loibner, A.P. (2005). Potential of preliminary test methods to predict biodegradation performance of petroleum hydrocarbon in soil. Biodegradation, 16:115125.

Alef, K. and Nanniperi, P. (1995). Methods in Applied Soil Microbiology and Biochemistry. Academic Press, San Diego, USA.

Amellal, N., Portal, J. M., Vogel, T., and Berthelin, J. (2001). Distribution and location of polycyclic aromatic hydrocarbons (PAHs) and PAH-degrading bacteria within polluted soil aggregates. Biodegradation, 12:49-57.

Bressler, D.C. and Gray, M.R. (2003). Transport and reaction processes in bioremediation of organic contaminants. 1. Review of bacterial degradation and transport. International Journal of Chemical Reactor Engineering, 1(1).

Brook, T.R., Stiver, W.H. and Zytner, R.G. (2001). Biodegradation of Diesel Fuel in Soil Under Various Nitrogen Addition Regimes, J. of Soil and Sediment Contamination, 10(5):539-553.

Canadian Council of Ministers of the Environment (CCME) (2008). Canadian Soil Quality Guidelines, Carcinogenic and other polycyclic aromatic hydrocarbons (PAHs). Scientific Supporting Document.

Chang, W., Whyte, L. and Ghoshal, S. (2011). Comparison of the effects of variable site temperatures and constant incubation temperatures on the biodegradation of petroleum hydrocarbons in pilotscale experiments with field-aged contaminated soils from a cold regions site. Chemosphere, 82:872-878.

Davis, C.A., Cort, T., Dai, D., Illangasekare, T.H. and Marr, J.M. (2003). Effects of heterogeneity and experimental scale on the biodegradation of diesel. Journal of Biodegradation, 14:373-384.

Del'Arco, J.P. and de Franca, F.P. (2001). Influence of oil contamination level on hydrocarbon biodegradation in sandy sediment. Environmental Pollution, 110:515-519.

El-Tarabily, K. A. (2002). Total microbial activity composition of a mangrove sediment are reduced by oil pollution at a site in the Arabian Gulf. Can. J. Microbiol. 48:176-182. 
Efroymson, M.A. (1960). Multiple regression analysis. In Ralston, A. and Wilf, HS, editors, Mathematical Methods for Digital Computers. Wiley.

Eyvazi, M.J. and Zytner, R.G. (2009). A Correlation to Estimate The Bioventing Rate Constant. Bioremediation Journal, 13(3):141-153.

Guo, H., Yao, J., Cai, M., Qian, Y., Guo, Y., Richnow, H.H., Blake, R.E., Doni, S. and Ceccanti, B. (2012). Effects of petroleum contamination on soil microbial numbers, metabolic activity and urease activity. Chemosphere, 87:1273-1280.

Harper, B., Stiver, W.H. and Zytner, R. G. (2003). Quantification of Water and NAPL Contents on SVE Contaminant Mass Transfer in Silt Loam Soil. ASCE J of Environmental Engineering. 129(8):745-755.

Harvey, H.R., Fallon, R.D. and Patton, J.S. (1986). The effect of organic matter and oxygen on the degradation of bacterial membrane lipids in marine sediments. Geochim. Cosmochim. Acta, 50:795-804.

Jiang, Y.F., Wang, X.T., Wu, M.H., Sheng, G.Y. and Fu, J.M. (2011). Contamination, source identification, and risk assessment of polycyclic aromatic hydrocarbons in agricultural soil of Shanghai, China. Environmental Monitor Assessment,183:139-150.

Khan, A.A. and Zytner, R.G. (2013). Degradation rates for petroleum hydrocarbons undergoing bioventing at the meso-scale. Bioremediation Journal, 17(3): 159-172.

Khan, F.I., Husain, T. and Hejazi, R. (2004). An overview and analysis of site remediation technologies. J. Environ. Management, 71:95-122.

Ko, I., Kim, K.W., Lee, C.H. and Lee, K.P. (2007). Effect of Scale-up and Seasonal Variation on Biokinetics in the Enhanced Bioremediation of Petroleum Hydrocarbon-contaminated Soil. Biotechnology and Bioprocess Engineering, 12:531-541.

Labud, V., Garcia, C. and Hernandez, T. (2007). Effect of hydrocarbon pollution on the microbial properties of a sandy and a clay soil. Chemosphere, 66:1863-1871.

Lee, T., Byun, I., Kim, Y., Hwang, I. and Park, T.J. (2006). Monitoring biodegradation of diesel fuel in bioventing processes using in situ respiration rate. Water Science Technol., 53(4-5):263-72. 
Leeson, A. and Hinchee, R. E. (1996). Principles and practices of bioventing volume I: bioventing principles. Battelle report prepared for the United States.

Maletić, S., Dalmacija, B., Rončević, S., Agbaba, J. and Petrović, O. (2009). Degradation Kinetics of an Aged Hydrocarbon-Contaminated Soil. Water Air and Soil Pollution, 202: 149-149.

McCarthy, K., Walker, L., Vigoren, L. and Bartel, J. (2004). Remediation of spilled petroleum hydrocarbons by in situ land farming at an Arctic site. Cold Region Science and Technology, 40:31-39.

Microsoft Excel (MSE) (2007). Excel Computer Software. Redmond, Washington.

Moller, J., Winther, P., Lund, B., Kirkebjerg, K. and Westermann, P. (1996). Bioventing of Diesel OilContaminated Soil: Comparison of Degradation Rate in Soil based on Actual Oil Concentration and respiration data. Journal of Industrial Microbiology, 16:110-116.

R Development Core Team (2013). R: A Language and Environment for Statistical Computing. software available at http://www.R-project.org, February.

Scully, N.M., Cooper, W.J. and Tranvik, L.J. (2003). Photochemical effects on microbial activity in natural waters: the interaction of reactive oxygen species and dissolved organic matter. FEMS Microbiology Ecology, 46:353-357.

Shewfelt, K., Lee, H. and Zytner, R.G. (2005). Optimization of nitrogen for bioventing of gasoline contaminated soil. Journal of Environmental Engineering, 4:29-42.

SYSTAT (1992). Statistical Analysis Package for Windows. Ver 5.02, SYSTAT Inc. Evanston, IL.

Trevors, J.T. (1996). Sterilization and inhibition of microbial activity in soil. Journal of Microbiological Methods, 26:53-59.

U.S. Environmental Protection Agency (USEPA) (2003). Aerobic Biodegradation of Oily Wastes, A Field Guidance Book For Federal On-scene Coordinators. Region 6 South Central Response and Prevention Branch. Version 1.0, October.

Wolf, D.C. and Skipper, H.D. (1994). Soil sterilization in: Methods of soil analysis part 2: Microbiological and Biochemical properties. Soil Sci. Soc. Amer., Madison, USA, 41-51. 
Yang, Y., Zhang, N., Xue, M., Lu, S.T. and Tao, S. (2011). Effects of soil organic matter on the development of the microbial polycyclic aromatic hydrocarbons (PAHs) degradation potentials. Environmental Pollution., 159:591-595.

Zytner, R.G., Salb, A.C., and Stiver, W.H. (2006). Bioremediation of diesel fuel contaminated soil: comparison of individual compounds to complex mixture of soil and sediment contamination. Soil Sediment Contamination: International Journal, 15(3):77-297.

Zytner, R.G., Salb, A., Brook, T. R., Leunissen, M. and Stiver, W. H. (2001). Bioremediation Of Diesel Fuel Contaminated Soil. Canadian Journal of Civil Engineering, 28:131-140. 


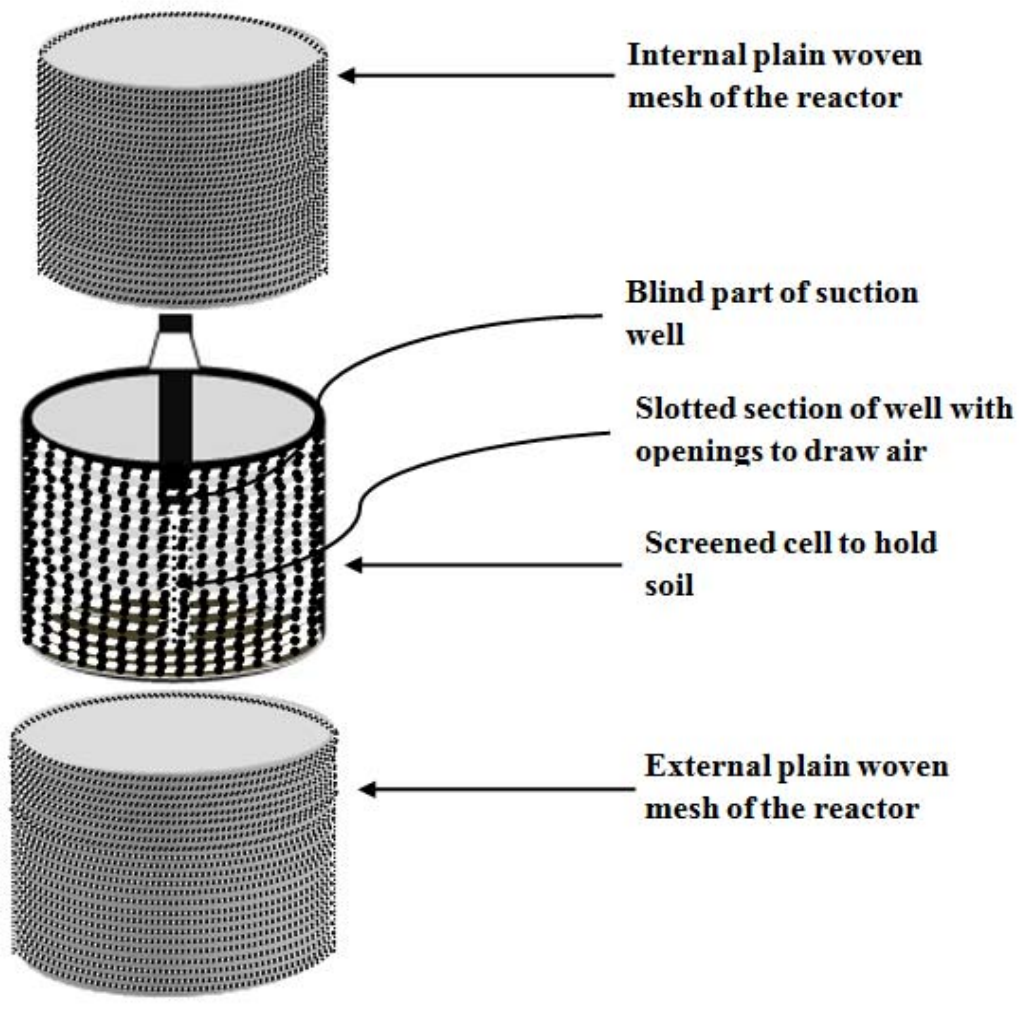

Figure 1: Sketch showing principal parts of meso-scale reactor 


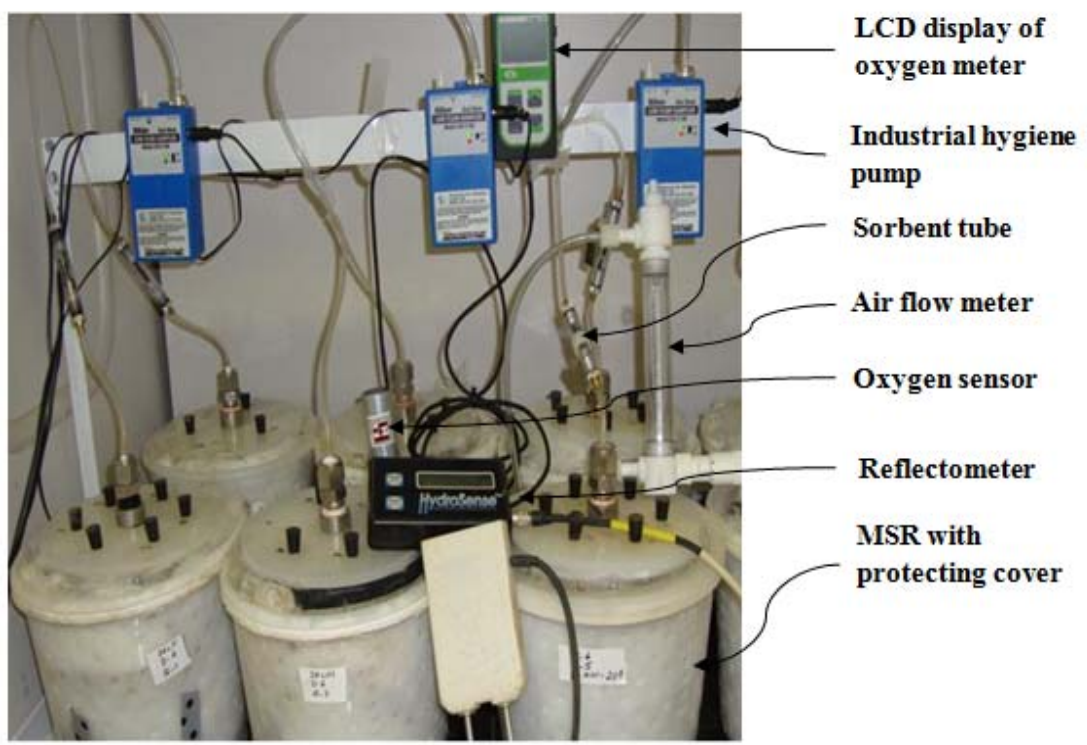

Figure 2: Experimental set up with Meso-Scale Reactor 


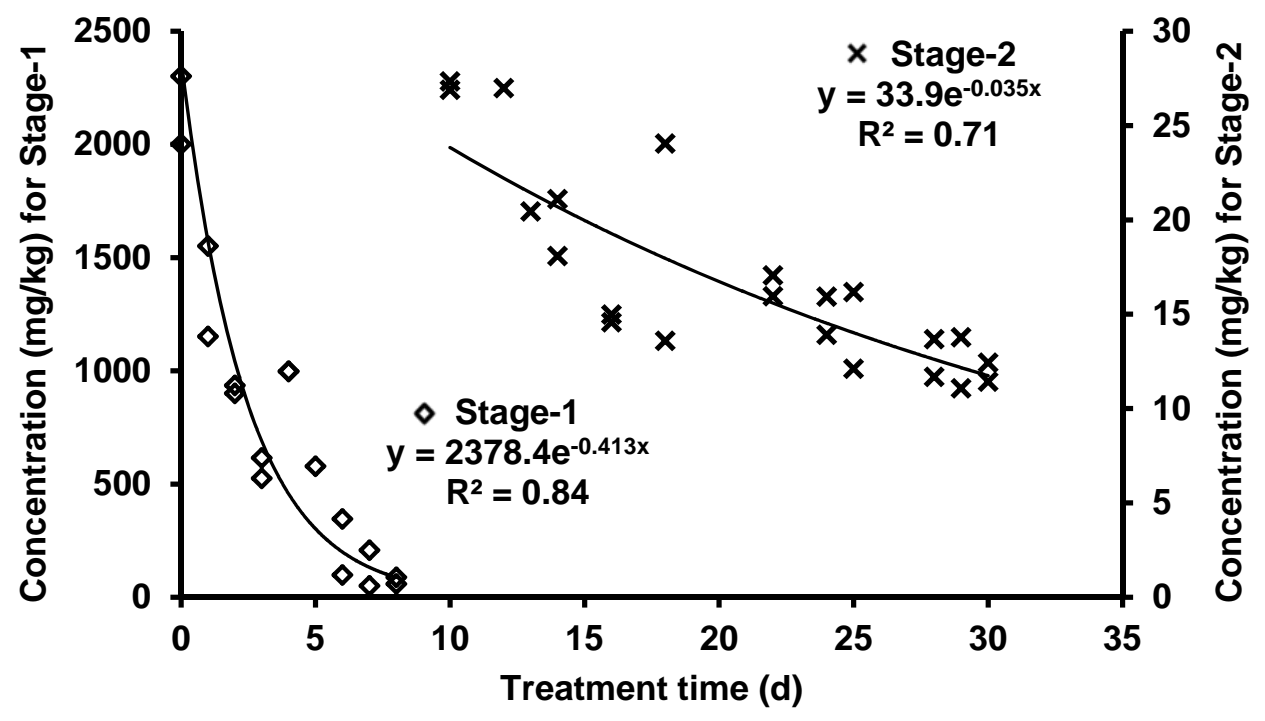

Figure 3: Degradation Trend of Synthetic Gasoline for pooled data of two treatments with RT-1 soil (Stage-1: 0-8d and Stage-2: 8-30d) 


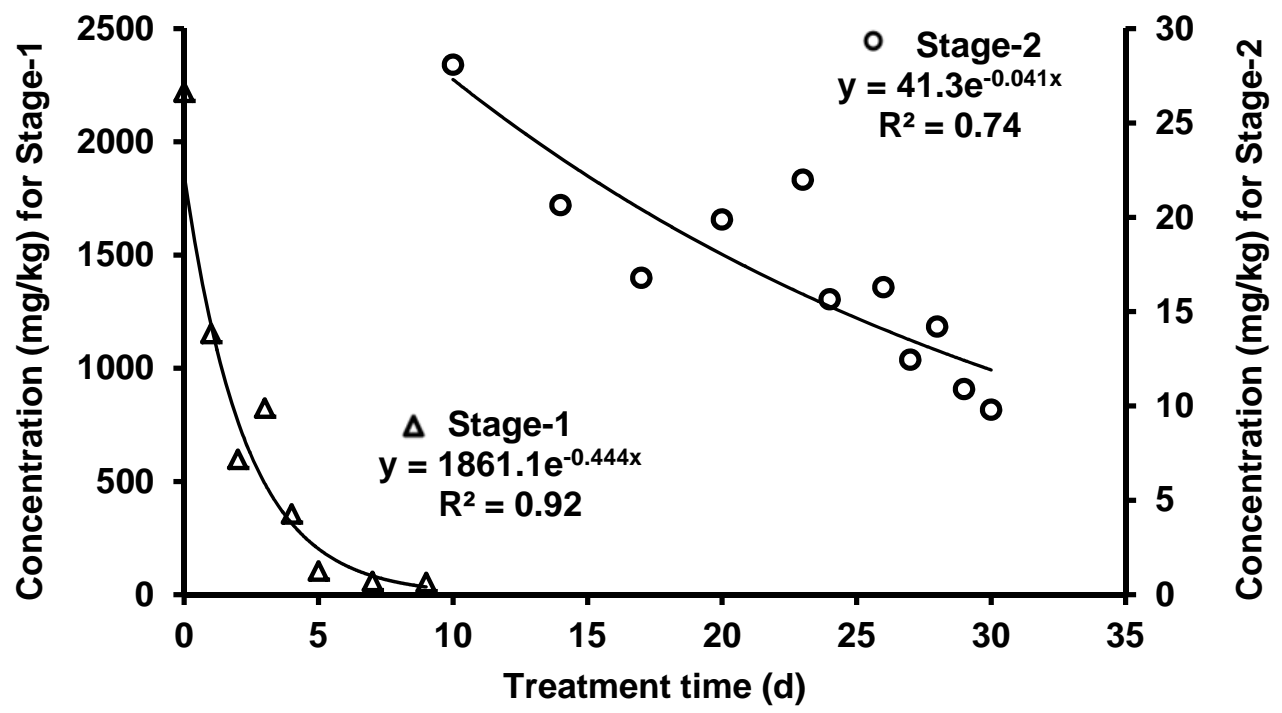

Figure 4: Degradation Trend of Synthetic Gasoline for RT-2 soil (Stage-1: 0-8d and Stage-2: 8-30d) 

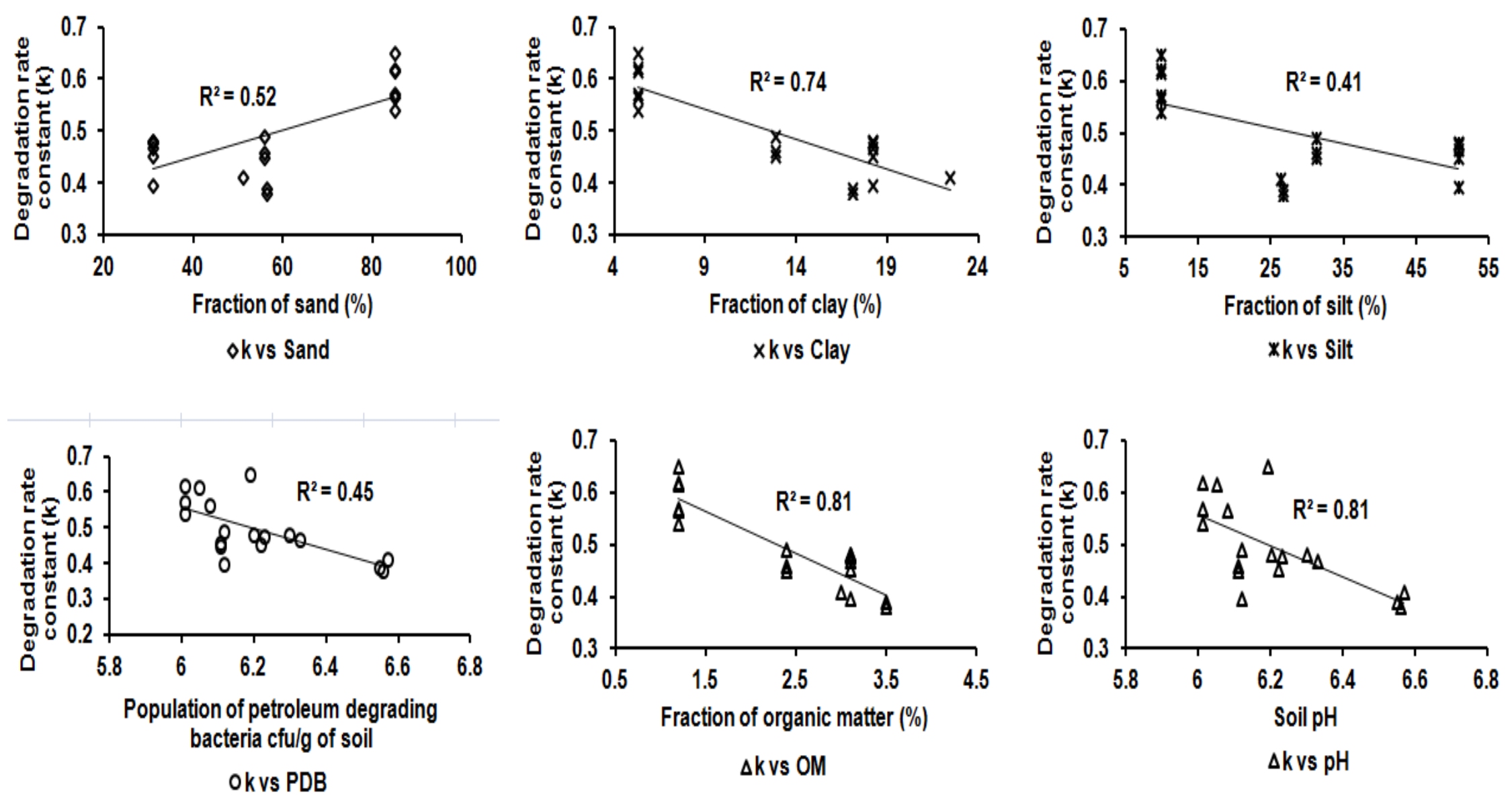

Figure 5: Relation of individual soil and biomass parameter with $k$ in Stage-1. 


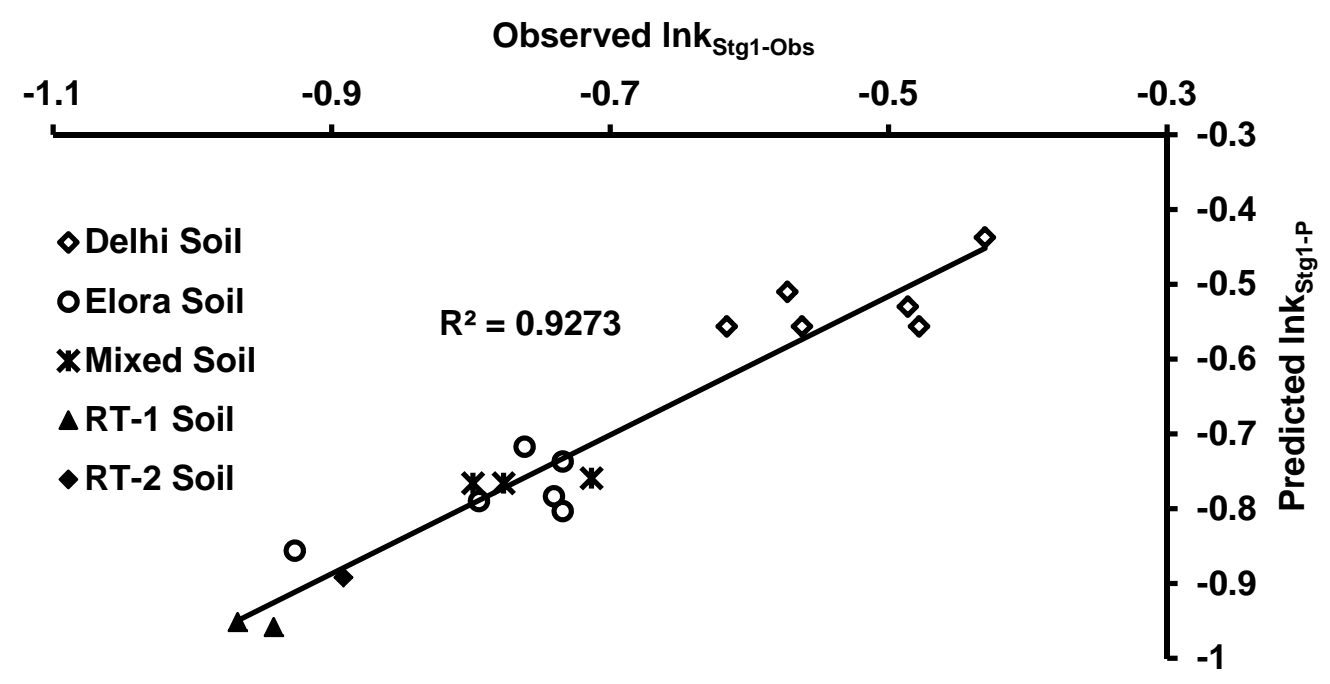

Figure 6: Plot of observed Ink $k_{S t g 1-O b s}$ against predicted $\ln _{\text {Stg1-P }}$ for Stage-1 


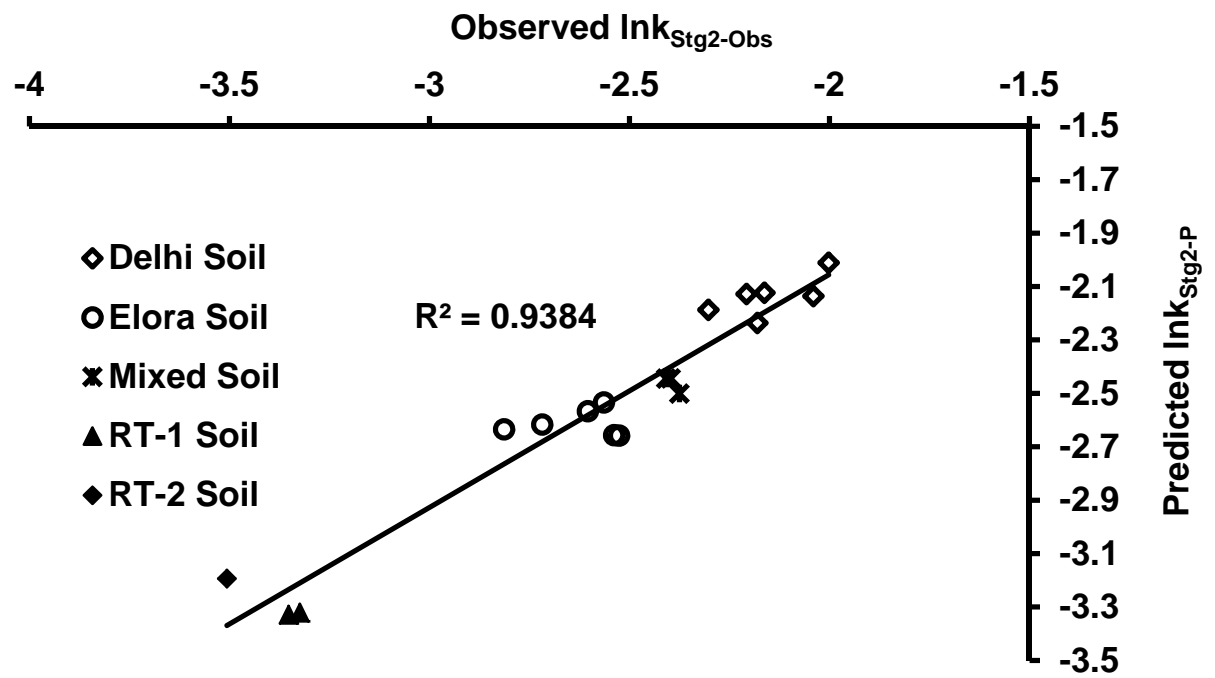

Figure 7: Plot of observed In $k_{\text {stg2-obs }}$ versus predicted $I n k_{\text {stg2-P }}$ for Stage-2 